\title{
COVERING THE SPHERE BY EQUAL ZONES
}

\author{
F. FODOR, V. VÍGH, AND T. ZARNÓCZ
}

\begin{abstract}
A zone of half-width $w$ on the unit sphere $S^{2}$ in Euclidean 3-space is a spherical segment of spherical width $2 w$ which is symmetric to $o$. L. Fejes Tóth raised the following question in [5]: what is the minimal $w_{n}$ such that one can cover $S^{2}$ with $n$ zones of width $2 w_{n}$ ? This question can be considered as a spherical relative of the famous plank problem of Tarski. We prove lower bounds for the minimum width $w_{n}$ for all $n \geq 5$.
\end{abstract}

\section{INTRODUCTION}

Let $S^{2}$ denote the unit sphere in 3 -dimensional Euclidean space $\mathbb{R}^{3}$ centred at the origin $o$. The spherical distance $d_{s}(x, y)$ of two points $x, y \in S^{2}$ is defined as the length of a (shorter) geodesic arc connecting $x$ and $y$ on $S^{2}$, or equivalently, the central angle $\angle x o y$ spanned by $x$ and $y$. Following L. Fejes Tóth [5], a zone $Z$ of half-width $w$ in $S^{2}$ is the parallel domain of radius $w$ of a great circle $C$, that is,

$$
Z(C, w):=\left\{x \in S^{2} \mid d_{s}(x, C) \leq w\right\} .
$$

We call $C$ the central great circle of $Z$. In this paper, we investigate the following problem.

Problem 1 (L. Fejes Tóth [5]). For a given n, find the smallest number $w_{n}$ such that one can cover $S^{2}$ with $n$ zones of half-width $w_{n}$. Find also the optimal configurations of zones that realize the optimal coverings.

We note that in the same paper [5] L. Fejes Tóth also asked the analogous question with not necessarily congruent zones, and conjectured that the sum of the widths of the zones that can cover $S^{2}$ is always at least $\pi$. Furthermore, L. Fejes Tóth [5] posed the question: what is the minimum of the sum of the widths of $n$ (not necessarily congruent) zones that can cover a spherically convex disc on $S^{2}$ ? These questions are similar to the classical plank problem of Tarski, see for example Bezdek [1] for a recent survey on this topic.

L. Fejes Tóth formulated the following conjecture:

Conjecture 1 (L. Fejes Tóth [5]). For $n \geq 1, w_{n}=\pi /(2 n)$.

It is clear that $w_{n} \leq \pi /(2 n)$ since $n$ zones of half-width $\pi /(2 n)$, whose central great circles all pass through a pair of antipodal points of $S^{2}$ and which are distributed evenly, cover $S^{2}$. On the other hand, as the zones must cover $S^{2}$, the sum of their areas must be at least (actually, greater than) $4 \pi$, that is, $w_{n}>\arcsin (1 / n)$.

Rosta [13] proved that $w_{3}=\pi / 6$, and that the unique optimal configuration consists of three zones whose central great circles pass through two antipodal points of $S^{2}$ and are distributed evenly. Linhart [9] showed that $w_{4}=\pi / 8$, and the unique optimal configuration is similar to the one for $n=3$. To the best of our knowledge, 
no further results about this problem have been achieved to date and thus L. Fejes Tóth's conjecture remains open.

The paper is organized as follows. In Section 2, we determine the area of the intersection of two congruent zones as a function of their half-widths and the angle of their central great circles under some suitable restrictions. In Section 3, we use the currently known best upper bounds for the maximum of the minimal pairwise spherical distances of $n$ points in $S^{2}$ to estimate from above the contribution of a zone in an optimal covering. Adding up these estimated contributions, we obtain a lower bound for $w_{n}$, which is the main result of our paper, and it is stated in Theorem 1. Finally, we calculate the numerical values of the established lower bound for some specific $n$.

\section{INTERSECTION OF TWO ZONES}

We start with the following simple observation. Consider two zones $Z_{1}$ and $Z_{2}$ of half-width $w$ whose central great circles make an angle $\alpha$. If $\alpha \geq 2 w$, then the intersection of $Z_{1}$ and $Z_{2}$ is the union of two disjoint congruent spherical domains. These domains are symmetric to each other with respect to $o$, and they resemble to a rhombus which is bounded by four small circular arcs of equal (spherical) length. If $\alpha \leq 2 w$, then the intersection is a connected, band-like domain. Let $2 F(w, \alpha)$ denote the area of $Z_{1} \cap Z_{2}$.

Lemma 1. Let $0 \leq w \leq \pi / 4$ and $2 w \leq \alpha \leq \pi / 2$. Then

$$
\begin{aligned}
F(w, \alpha)=2 \pi & +4 \sin w \arcsin \left(\frac{1-\cos \alpha}{\cot w \sin \alpha}\right)+4 \sin w \arcsin \left(\frac{1+\cos \alpha}{\cot w \sin \alpha}\right) \\
& -2 \arccos \left(\frac{\cos \alpha-\sin ^{2} w}{\cos ^{2} w}\right)-2 \arccos \left(\frac{-\cos \alpha-\sin ^{2} w}{\cos ^{2} w}\right) .
\end{aligned}
$$

Moreover, $F(w, \alpha)$ is a monotonically decreasing function of $\alpha$ in the interval $[0, \pi / 2]$.

Proof. First, we prove (1). Let $Z_{1}$ be the zone of half-width $w$ whose central great circle $C_{1}$ is the intersection of the $x y$-plane with $S^{2}$. Let $c_{1}$ and $c_{3}$ denote the small circles which bound $Z_{1}$ such that $c_{1}$ is contained in the closed half-space $z \geq 0$.

Let $Z_{2}$ be the zone of half-width $w$ whose central great circle $C_{2}$ is the intersection of $S^{2}$ with the plane which contains the $y$-axis and which makes an angle $\alpha$ with the $x y$-plane as shown in Figure 1 . Let $c_{2}$ and $c_{4}$ be the small circles bounding $Z_{2}$, cf. Figure 1.

The intersection $Z_{1} \cap Z_{2}$ is the union of two connected components $R_{1}$ and $R_{2}$. Assume that $R_{1}$ is contained in the closed half-space $y \leq 0$. Let $c_{i}^{\prime}, i=1, \ldots, 4$ denote the arc of $c_{i}$ that bounds $R_{1}$. Observe that the $c_{i}^{\prime}$ are of equal length; we denote their common arc length by $l(w, \alpha)$. The radii of $c_{1}, \ldots, c_{4}$ are all equal to $\cos w$.

Assume that the boundary $\partial R_{1}$ of $R_{1}$ is oriented such that the small circular arcs follow each other in the cyclic order $c_{1}^{\prime}, c_{2}^{\prime}, c_{3}^{\prime}, c_{4}^{\prime}$. For $i \in\{1, \ldots, 4\}$, let $\varphi_{i}(w, \alpha)$ denote the turning angle of $\partial R_{1}$ at the intersection point of $c_{i}^{\prime}$ and $c_{i+1}^{\prime}$ with the convention that $c_{5}=c_{1}$. Notice that the signed geodesic curvature of $\partial R_{1}$ (in its smooth points) is equal to $-\tan w$. 


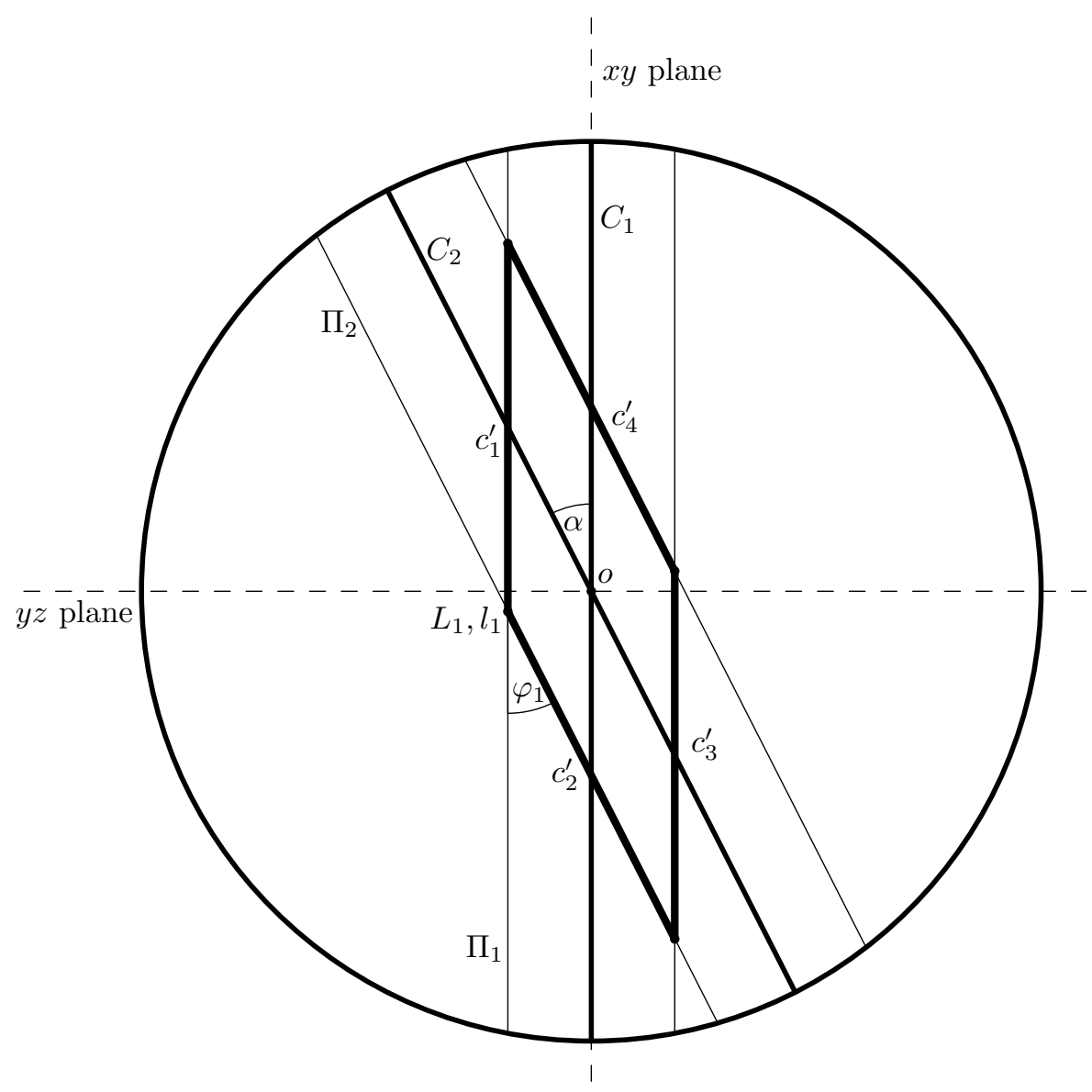

Figure 1. Orthogonal projection onto the $x z$ plane

By the Gauss-Bonnet Theorem it holds that

$$
F(w, \alpha)=2 \pi+4 \tan w \cdot l(w, \alpha)-\sum_{i=1}^{4} \varphi_{i}(w, \alpha)
$$

Next, we calculate the $\varphi_{i}(w, \alpha)$. Note that $\varphi_{i}(w, \alpha)=\varphi_{i+2}(w, \alpha)$ for $i=1,2$.

Let $\Pi_{1}$ be the plane whose normal vector is $u_{1}=(0,0,1)$ and contains the point $(0,0, \sin w)$. Let $\Pi_{2}$ be the plane which we get by rotating $\Pi_{1}$ around the $y$-axis by angle $\alpha$ so its normal vector is $u_{2}=(-\sin \alpha, 0, \cos \alpha)$, see Figure 1 . Note that $S^{2} \cap \Pi_{1}=c_{1}$ and $S^{2} \cap \Pi_{2}=c_{2}$.

$$
\begin{aligned}
& \Pi_{1}: \quad z=\sin w \\
& \Pi_{2}: \quad-x \sin \alpha+z \cos \alpha=\sin w
\end{aligned}
$$


Now let $L_{1}=\Pi_{1} \cap \Pi_{2}$ and $L_{1} \cap S^{2}=\left\{l_{1}, l_{1}^{\prime}\right\}$, such that $l_{1}$ has negative $y$-coordinate. Then

$$
l_{1}=\left(\sin w(\cot \alpha-\csc \alpha),-\sqrt{1-\sin ^{2} w\left(1+(\cot \alpha-\csc \alpha)^{2}\right)}, \sin w\right) .
$$

Let $\Pi$ be the plane that is tangent to $S^{2}$ in $l_{1}$, and let $E_{1}=\Pi_{1} \cap \Pi$ and $E_{2}=\Pi_{2} \cap \Pi$. Then $\varphi_{1}$ is one of the angles made by $E_{1}$ and $E_{2}$. Let $v_{1}=l_{1} \times u_{1}$ and $v_{2}=l_{1} \times u_{2}$. Then $v_{1}$ and $v_{2}$ are vectors parallel to $E_{1}$ and $E_{2}$, respectively, such that their orientations agree with that of $\partial R_{1}$.

$$
\begin{aligned}
& v_{1}=\left(-\sqrt{1-\sin ^{2} w\left(1+(\cot \alpha-\csc \alpha)^{2}\right)},-\sin w(\cot \alpha-\csc \alpha), 0\right) \\
& v_{2}=\left(-\cos \alpha \sqrt{1-\sin ^{2} w\left(1+(\cot \alpha-\csc \alpha)^{2}\right)},-\cos \alpha \sin w(\cot \alpha-\csc \alpha)-\right. \\
& \left.-\sin \alpha \sin w,-\sin \alpha \sqrt{1-\sin ^{2} w\left(1+(\cot \alpha-\csc \alpha)^{2}\right)}\right) .
\end{aligned}
$$

We only need to calculate the lengths of $v_{1}$ and $v_{2}$ and their scalar product. By routine calculations we obtain

$$
\varphi_{1}=\arccos \frac{\left\langle v_{1}, v_{2}\right\rangle}{\left|v_{1}\right|\left|v_{2}\right|}=\arccos \left(\frac{\cos \alpha-\sin ^{2} w}{\cos ^{2} w}\right) .
$$

The angle $\varphi_{2}$ can be evaluated similarly; one only needs to write $\pi-\alpha$ in place of $\alpha$ in the above calculations. Then

$$
\varphi_{2}=\arccos \left(\frac{-\cos \alpha-\sin ^{2} w}{\cos ^{2} w}\right) .
$$

To finish the calculation, we need to find $l(w, \alpha)$. Let $l_{i}:=c_{i}^{\prime} \cap c_{i+1}^{\prime}$ for $i=1,2,3,4$ with $c_{5}^{\prime}=c_{1}^{\prime}$. Let $d_{i}, i=1, \ldots, 4$ be the absolute value of the $y$-coordinate of $l_{i}$. Simple trigonometry shows that

and

$$
d_{1}=\frac{1-\cos \alpha}{\cot w \sin \alpha}
$$

$$
d_{4}=\frac{1+\cos \alpha}{\cot w \sin \alpha}
$$

Then the length of $c_{1}^{\prime}$ is equal to the following

$$
\begin{aligned}
l(w, \alpha) & =\cos w \arcsin d_{1}+\cos w \arcsin d_{4} \\
& =\cos w \arcsin \left(\frac{1-\cos \alpha}{\cot w \sin \alpha}\right)+\cos w \arcsin \left(\frac{1+\cos \alpha}{\cot w \sin \alpha}\right) .
\end{aligned}
$$

In summary,

$$
\begin{aligned}
F(w, \alpha)=2 \pi & +4 \sin (w) \arcsin (\tan (w)(\csc (\alpha)+\cot (\alpha))) \\
& +4 \sin (w) \arcsin (\tan (w)(\csc (\alpha)-\cot (\alpha))) \\
& -2 \arccos \left(\frac{\cos (\alpha)-\sin ^{2}(w)}{\cos ^{2}(w)}\right) \\
& -2 \arccos \left(\frac{\cos (\alpha)+\sin ^{2}(w)}{-\cos ^{2}(w)}\right)
\end{aligned}
$$

Finally, we prove that $F$ is monotonically decreasing in $\alpha$. This is obvious in the interval $[0,2 w]$. 
Let $\varepsilon>0$ be sufficiently small with $\alpha+\varepsilon \leq \pi / 2$. Consider the spherical "rhombus" $R_{1}^{*}$ which is obtained as the intersection of $Z_{1}$ and another zone $Z_{2}^{*}$ of halfwidth $w$ whose central great circle $C_{2}^{*}$ is the intersection of $S^{2}$ with the plane which contains the $y$-axis and which makes an angle $\alpha+\varepsilon$ with the $x y$-plane, similarly as for $Z_{2}$ above. Let $F_{1}$ be the area of $R_{1} \backslash R_{1}^{*}$ and $F_{1}^{*}$ be the area of $R_{1}^{*} \backslash R_{1}$. For the monotonicity of $F(w, \alpha)$ in $\alpha$, we only need to show that $F_{1}>F_{1}^{*}$.

The region $R_{1} \backslash R_{1}^{*}$ consists of two disjoint congruent connected domains (in fact, two triangular regions bounded by arcs of small circles). Note that one such region, say $P$, is fully contained in the positive hemisphere of $S^{2}(z \geq 0)$, and the other region is contained in the negative hemisphere $(z \leq 0)$. Similarly, let $Q$ be the one of the two connected, congruent and disjoint regions whose union is $R_{1}^{*} \backslash R_{1}$ and which has a common (boundary) point with $P$. Let $q=P \cap Q$, then $q$ has positive $z$-coordinate. It easily follows from the position of $q$ that the $\operatorname{arc} c_{2} \cap Q$ is longer than $c_{2} \cap P$, and, similarly, $c_{2}^{*} \cap Q$ is longer than $c_{2}^{*} \cap P$, so the area of $Q$ is larger than the area of $P$, which completes the proof of the Lemma.

Remark 1. Let $Z_{1}$ and $Z_{2}$ be two zones of half-width $w \in(0, \pi / 4]$ which make an angle $\alpha$. Then it is clear that the area of $Z_{1} \cup Z_{2}$ is a monotonically increasing function of $\alpha$ for $\alpha \in[0,2 w]$.

\section{A LOWER BOUND FOR $w_{n}$}

For an integer $n \geq 3$, let $d_{n}$ denote the maximum of the minimal pairwise (spherical) distances of $n$ points on the unit sphere $S^{2}$. Finding $d_{n}$ is a long-standing problem of discrete geometry which goes back to the Dutch botanist Tammes (1930) (see [15]). As of now, the exact value of $d_{n}$ is only known in the following cases.

\begin{tabular}{|c|c|c|}
\hline$n$ & $d_{n}$ & \\
\hline 3 & $2 \pi / 3$ & L. Fejes Tóth [7] \\
\hline 4 & 1.91063 & L. Fejes Tóth [7] \\
\hline 5 & $\pi / 2$ & Schütte, van der Waerden [14] \\
\hline 6 & $\pi / 2$ & L. Fejes Tóth [7] \\
\hline 7 & 1.35908 & Schütte, van der Waerden [14] \\
\hline 8 & 1.30653 & Schütte, van der Waerden [14] \\
\hline 9 & 1.23096 & Schütte, van der Waerden [14] \\
\hline 10 & 1.15448 & Danzer [4] \\
\hline 11 & 1.10715 & Danzer [4] \\
\hline 12 & 1.10715 & L. Fejes Tóth [7] \\
\hline 13 & 0.99722 & Musin, Tarasov [11] \\
\hline 14 & 0.97164 & Musin, Tarasov [10] \\
\hline 24 & 0.76255 & Robinson [12] \\
\hline
\end{tabular}

TABLE 1. Known values of $d_{n}$

Alternate proofs were given by Hárs [8] for the case $n=10$, and by Böröczky [2] for the case $n=11$. 
For $n \geq 3$, L. Fejes Tóth (see [6]) proved the following upper estimate

$$
d_{n} \leq \tilde{\delta}_{n}:=\arccos \left(\frac{\cot ^{2}\left(\frac{n}{n-2} \frac{\pi}{6}\right)-1}{2}\right),
$$

where equality holds exactly in the cases $n=3,4,6,12$ (see table above). Moreover, $\lim _{n \rightarrow \infty} \tilde{\delta}_{n} / d_{n}=1$, that is, $\tilde{\delta}_{n}$ provides an exact asymptotic upper bound for $d_{n}$ as $n \rightarrow \infty$.

Robinson [12] improved the upper estimate (4) of L. Fejes Tóth as follows. Assume that the pairwise distances between the $n$ points on the sphere are all at least $a$ where $0<a<\arctan 2$. Let $\Delta_{1}(a)$ denote the area of an equilateral spherical triangle with side lengths $a$, and $\Delta_{2}(a)$ denote the area of a spherical triangle with two sides of length $a$ making an angle of $2 \pi-4 \alpha$. Let $\delta_{n}$ be the unique solution of the equation $4 n \Delta_{1}(a)+(2 n-12) \Delta_{2}(a)-12 \pi=0$. Then (cf. [12]) $d_{n} \leq \delta_{n} \leq \tilde{\delta}_{n}$ for $n \geq 13$.

Let $d_{n}^{*}:=\min \left\{\pi / 2, d_{n}\right\}$ for $n \geq 2$, and let

$$
\delta_{n}^{*}:= \begin{cases}d_{n}^{*} & \text { for } 3 \leq n \leq 14 \text { and } n=24, \\ \delta_{n} & \text { otherwise. }\end{cases}
$$

We will also need a lower bound on $d_{n}$ for our argument. We note that, for example, van der Waerden [16] proved a non-trivial lower bound on $d_{n}$, however, for our purposes the following simpler bound is sufficient. Set $\varrho_{n}:=\arccos (1-2 / n)$, and consider a maximal (saturated) set of points $p_{1}, \ldots, p_{m}$ on the unit sphere $S^{2}$, such that their pairwise spherical distances are at least $\varrho_{n}$. By maximality it follows that the spherical circular discs (spherical caps) of radius $\varrho_{n}$ centered at $p_{1}, \ldots, p_{m}$ cover $S^{2}$. As the (spherical) area of such a cap is $4 \pi / n$, we obtain that $m \cdot 4 \pi / n \geq 4 \pi$, that is, $m \geq n$, which implies that $\varrho_{n}:=\arccos (1-2 / n) \leq d_{n}$. As $x \leq \arccos \left(1-x^{2} / 2\right)$ for $0 \leq x \leq 1$, the following inequality is immediate

$$
\frac{2}{\sqrt{n}} \leq d_{n}^{*} \leq \delta_{n}^{*}
$$

For $0 \leq \alpha \leq \pi / 2$ and $n \geq 3$ we introduce $f(w, \alpha)=4 \pi \sin w-2 F(w, \alpha)$ and

$$
G(w, n)=4 \pi \sin w+\sum_{i=2}^{n} f\left(w, \delta_{2 i}^{*}\right) .
$$

Lemma 2. For a fixed $n \geq 3$, the function $G(w, n)$ is continuous and monotonically increasing in $w$ in the interval $\left[0, \delta_{2 n}^{*} / 3\right]$. Furthermore, $G(0, n)=0$ and $G\left(\delta_{2 n}^{*} / 3, n\right) \geq 4 \pi$.

Proof. The continuity of $G$ and that $G(0, n)=0$ are obvious. First we show that the function $f(w, \alpha)$ is monotonically increasing in $w$ for $0 \leq w \leq \alpha / 3$. This clearly implies that $G(w, n)$ is also monotonically increasing in the interval stated in the lemma. As $n \geq 3$, we may and do assume that $w \leq \delta_{6}^{*} / 3=\pi / 6$.

Note that $f(w, \alpha)$ is the area of a zone of half-width $w$ minus the area of its intersection with a second zone of half-width $w$ whose central great circle makes an angle $\alpha$ with the central great circle of the first zone. With the same notations as in the proof of Lemma 1, it is clear that for sufficiently small $\Delta w>0$, the quantity $f(w+\Delta w, \alpha)-f(w, \alpha)$ is (roughly) proportional to $2 l\left(c_{1}\right)-4 l\left(c_{1}^{\prime}\right)-4 l\left(c_{2}^{\prime}\right)=$ 
$2\left(l\left(c_{1}\right)-4 l\left(c_{1}^{\prime}\right)\right)$. Notice that, for a fixed $w \in[0, \pi / 4]$, the function $l\left(c_{1}^{\prime}\right)=l(w, \alpha)$ is monotonically decreasing in $\alpha$ for $\alpha \in[2 w, \pi / 2]$. Thus, using $3 w \leq \alpha$,

$$
\begin{aligned}
l\left(c_{1}\right)-4 l\left(c_{1}^{\prime}\right) & \geq l\left(c_{1}\right)-4 l(w, 3 w)= \\
& =4 \cos w\left(\frac{\pi}{2}-\arcsin \left(\frac{1-\cos (3 w)}{\cot w \sin (3 w)}\right)-\arcsin \left(\frac{1+\cos (3 w)}{\cot w \sin (3 w)}\right)\right) .
\end{aligned}
$$

One can check that if $w \in(0, \pi / 6]$, then both arguments in the above arcsin functions take on values in $[0,2 / 3]$. By the monotonicity and convexity of arcsin, we obtain that

$$
\begin{aligned}
& \arcsin \left(\frac{1-\cos (3 w)}{\cot w \sin (3 w)}\right)+\arcsin \left(\frac{1+\cos (3 w)}{\cot w \sin (3 w)}\right) \leq \arcsin (2 / 3) \frac{3 \tan w}{\sin (3 w)} \\
& \leq \arcsin (2 / 3) \frac{3 \tan (\pi / 6)}{\sin (\pi / 2)}=\frac{2 \sqrt{3}}{3}<\frac{\pi}{2},
\end{aligned}
$$

which shows the monotonicity of $G(w, n)$.

Finally, we show that $G\left(\delta_{2 n}^{*} / 3, n\right) \geq 4 \pi$. For $n \leq 24$, this statement can be checked by direct calculation, thus we may assume $n \geq 25$. Using the definitions of $G$ and $f$, and Lemma 1 , we obtain that

$$
\begin{aligned}
G\left(\frac{\delta_{2 n}^{*}}{3}, n\right) & =n \cdot 4 \pi \sin \frac{\delta_{2 n}^{*}}{3}-2 \cdot \sum_{i=2}^{n} F\left(\frac{\delta_{2 n}^{*}}{3}, \delta_{2 i}^{*}\right) \\
& \geq 4 n \pi \sin \frac{\delta_{2 n}^{*}}{3}-2 \sum_{i=2}^{n} F\left(\frac{\delta_{2 n}^{*}}{3}, \delta_{2 n}^{*}\right) \\
& =4 n \pi \sin \frac{\delta_{2 n}^{*}}{3}-2(n-1) F\left(\frac{\delta_{2 n}^{*}}{3}, \delta_{2 n}^{*}\right) \\
& \geq 4 n \pi \sin \frac{\delta_{2 n}^{*}}{3}-2(n-1) F\left(\frac{\delta_{2 n}^{*}}{3}, \frac{2 \delta_{2 n}^{*}}{3}\right) .
\end{aligned}
$$

Note that $\delta_{2 n}^{*}=\delta_{2 n}$ for $n \geq 25$. Elementary trigonometry yields that

$$
F\left(\frac{\alpha}{2}, \alpha\right)=4 \sin \frac{\alpha}{2} \arcsin \left(\tan ^{2} \frac{\alpha}{2}\right)+2 \pi \sin \frac{\alpha}{2}-2 \arccos \left(1-2 \tan ^{2} \frac{\alpha}{2}\right) .
$$

Thus (7) is equal to

$$
4 \pi \sin \frac{\delta_{2 n}}{3}+4(n-1)\left(\arccos \left(1-2 \tan ^{2} \frac{\delta_{2 n}}{3}\right)-2 \sin \frac{\delta_{2 n}}{3} \arcsin \left(\tan ^{2} \frac{\delta_{2 n}}{3}\right)\right) .
$$

As $n \geq 25$, we have that $0<\delta_{2 n}<0.75$. Using that $\cos x \geq 1-x^{2} / 2$ for $x \in[0, \pi / 2]$, we obtain that

$$
\arccos \left(1-2 \tan ^{2} \frac{\delta_{2 n}}{3}\right) \geq 2 \tan \frac{\delta_{2 n}}{3} .
$$

Similarly, as for $0<x<0.16$ we have that $x<1.01 \sin x$, we obtain that

$$
2 \sin \frac{\delta_{2 n}}{3} \arcsin \left(\tan ^{2} \frac{\delta_{2 n}}{3}\right)<2.02 \tan ^{3} \frac{\delta_{2 n}}{3} .
$$

Finally, using that $x-1.01 x^{3}>x-1.01 \cdot 0.4^{2} \cdot x>0.8 x$ for $0<x<0.4$, we obtain that $(7)$ can be estimated from below as follows

$$
G\left(\frac{\delta_{2 n}^{*}}{3}, n\right) \geq 6.4(n-1) \tan \frac{\delta_{2 n}}{3}>2.1(n-1) \delta_{2 n} .
$$


By (6) we know that $\delta_{2 n}>\sqrt{2} / \sqrt{n}$, and thus the proof of Lemma 2 is complete.

Now, we are ready to state our main theorem.

Theorem 1. For $n \geq 3$, let $w_{n}^{*}$ denote the unique solution of the equation $G(w, n)=4 \pi$ in the interval $\left[0, \delta_{2 n}^{*} / 3\right]$. Then $\arcsin (1 / n)<w_{n}^{*} \leq w_{n}$.

Proof. Let $Z_{i}\left(w_{n}, C_{i}\right), i=1, \ldots, n$ be zones that form a (minimal with respect to $w)$ covering of $S^{2}$. For $i \in\{1, \ldots, n\}$, let $p_{i}$ be one of the poles of $C_{i}$ and let $p_{n+i}=-p_{i}$. Then there exist two points $p_{i_{1}}, p_{j_{1}} \in\left\{p_{1}, \ldots, p_{2 n}\right\}$ with $i_{1}<j_{1}$ and $j_{1} \neq n+i_{1}$ (that is, $p_{i_{1}}$ and $p_{j_{1}}$ are poles of two different great circles) such that $d_{s}\left(p_{i_{1}}, p_{j_{1}}\right) \leq d_{2 n}^{*}$. Observe that the area of the part of $Z_{i_{1}}$ that is not covered by any $Z_{k}$ with $i_{1} \neq k$ is at most $f\left(w, \delta_{2 n}^{*}\right)$ by Lemma 1 , inequality (6) and Remark 1. Now, remove $Z_{i_{1}}$ from the covering and repeat the argument for the remaining zones. Note that in the last step of the process, there is only one zone left $Z_{i_{n}}$, so the area of the part of $Z_{i_{n}}$ not covered by any other zone is $4 \pi \sin w$.

If for $k=1, \ldots, n$ we add the areas of $Z_{i_{k}}$ not covered by any $Z_{i_{l}}$ for $l>k$, we obtain the function $G(w, n)$. Since $Z_{1}, \ldots, Z_{n}$ cover $S^{2}$, therefore $G(w, n) \geq 4 \pi$, which shows that $w_{n}^{*} \leq w_{n}$. It is also clear form the argument that $\arcsin (1 / n)<$ $w_{n}^{*}$. This finishes the proof of Theorem 1 .

\section{Concluding Remarks}

Remark 2. Instead of Robinson's bound $\delta_{n}$, one may use the original bound $\tilde{\delta}_{n}$ of L. Fejes Tóth, and prove Theorem 1, obtaining a lower bound $\tilde{w}_{n}^{*}$ for $w_{n}$. Clearly, this bound is slightly weaker than $w_{n}^{*}$, that is, $\tilde{w}_{n}^{*} \leq w_{n}^{*} \leq w_{n}$. However, we note that, thanks to the explicit formula (4), $\tilde{w}_{n}^{*}$ can be computed more easily than $w_{n}^{*}$. The difference between $w_{n}^{*}$ and $\tilde{w}_{n}^{*}$ is shown in Table 2 for some specific values of $n$.

We also mention that for certain values of $n$ Robinson's upper bound has been improved, see for example Böröczky and Szabó [3] for the cases $n=15,16,17$. These stronger upper bounds, if included in the calculations, would provide only a very small improvement on $w_{n}^{*}$, so we decided to use only the known solutions of the Tammes problem and Robinson's general upper bound.

Remark 3. We note that the analogous question to Problem 1 can be raised in higher dimensions as well. A zone $Z=Z(C, w)$ of half-width $w$ on the unit sphere $S^{d-1}$ of the $d$-dimensional Euclidean space $\mathbb{R}^{d}$ is the parallel domain of radius $w$ a great sphere $C$. What is the mininal $w(d, n)$ such that one can cover $S^{d-1}$ with $n$ zones of half-width $w(d, n)$, and what configurations realize the optimal coverings? We do not wish to formulate a conjecture about this problem, instead, we note the following simple fact. For $d \geq 4, w(d, 3)=\pi / 6$. One can see this the following way. Let $Z_{i}=Z\left(C_{i}, w\right), i=1,2,3$ be three zones that cover $S^{d-1}$. Assume that $C_{i}=S^{d-1} \cap H_{i}$ for $i=1,2,3$ where $H_{i}$ is a hyperplane. Let $L=\cap_{i} H_{i}$. Then $L$ is a linear subspace of $\mathbb{R}^{d}$, and $\operatorname{dim} L \geq d-3$. Let $L^{\perp}$ denote the linear subspace of $\mathbb{R}^{d}$ which is the orthogonal complement of $L$. Clearly, $L^{\perp} \cap S^{d-1}=S^{j}$, where $j \leq 2$. If $\operatorname{dim} L^{\perp}=1$, then $w=\pi / 2$. So we may assume that $\operatorname{dim} L^{\perp}=2$ or 3. Notice that the zones $Z_{i}, i=1,2,3$ cover $S^{d-1}$ if and only if the zones $Z_{i}^{\prime}=Z_{i} \cap\left(L^{\perp} \cap S^{d-1}\right), i=1,2,3$ cover $L^{\perp} \cap S^{d-1}=S^{j}$. We note also that the half-widths of $Z_{i}^{\prime}, i=1,2,3$ are all equal to $w$. Now, if $j=1$, then it is clear that 


\begin{tabular}{|c|c|c|c|c|}
\hline$n$ & $\arcsin (1 / n)$ & $\tilde{w}_{n}^{*}$ & $w_{n}^{*}$ & $\pi /(2 n)$ \\
\hline 5 & 0.20135 & 0.22983 & 0.22983 & 0.31415 \\
\hline 6 & 0.16744 & 0.18732 & 0.18732 & 0.26179 \\
\hline 7 & 0.14334 & 0.15824 & 0.15824 & 0.22439 \\
\hline 8 & 0.12532 & 0.13692 & 0.13692 & 0.19634 \\
\hline 9 & 0.11134 & 0.12063 & 0.12067 & 0.17453 \\
\hline 10 & 0.10016 & 0.10782 & 0.10787 & 0.15707 \\
\hline 11 & 0.09103 & 0.09748 & 0.09753 & 0.14279 \\
\hline 12 & 0.08343 & 0.08895 & 0.08899 & 0.13089 \\
\hline 13 & 0.07699 & 0.08179 & 0.08183 & 0.12083 \\
\hline 14 & 0.07148 & 0.07569 & 0.07573 & 0.11219 \\
\hline 15 & 0.06671 & 0.07044 & 0.07048 & 0.10471 \\
\hline 16 & 0.06254 & 0.06587 & 0.06591 & 0.09817 \\
\hline 17 & 0.05885 & 0.06185 & 0.06189 & 0.09239 \\
\hline 18 & 0.05558 & 0.05830 & 0.05833 & 0.08726 \\
\hline 19 & 0.05265 & 0.05513 & 0.05516 & 0.08267 \\
\hline 20 & 0.05002 & 0.05229 & 0.05232 & 0.07853 \\
\hline 21 & 0.04763 & 0.04972 & 0.04975 & 0.07479 \\
\hline 22 & 0.04547 & 0.04740 & 0.04743 & 0.07139 \\
\hline 23 & 0.04349 & 0.04528 & 0.04531 & 0.06829 \\
\hline 24 & 0.04167 & 0.04335 & 0.04337 & 0.06544 \\
\hline 25 & 0.04001 & 0.04157 & 0.04159 & 0.06283 \\
\hline 50 & 0.02000 & 0.02050 & 0.02051 & 0.03141 \\
\hline 100 & 0.01000 & 0.01016 & 0.01017 & 0.01570 \\
\hline & &
\end{tabular}

TABLE 2. Bounds for $w_{n}$

$w \geq \pi / 6$ by elementary geometry, and if $j=2$, then by Rosta's result [13], it holds that $w \geq w_{3}=\pi / 6$. Finally, in both cases, $w=\pi / 6$ suffices to cover $S^{d-1}$.

\section{Acknowledgements}

The authors wish to thank András Bezdek for the useful discussions about the problem.

This paper was supported in part by TÁMOP-4.2.2.B-15/1/KONV-2015-0006. F. Fodor wishes to thank the MTA Alfréd Rényi Institute of Mathematics of the Hungarian Academy of Sciences, where part of this work was done while he was a visiting researcher. V. Vígh was supported by the János Bolyai Research Scholarship of the Hungarian Academy of Sciences.

\section{REFERENCES}

[1] K. Bezdek, Tarski's plank problem revisited, Geometry-intuitive, discrete, and convex, Bolyai Soc. Math. Stud., vol. 24, János Bolyai Math. Soc., Budapest, 2013, pp. 45-64.

[2] K. Böröczky, The problem of Tammes for $n=11$, Studia Sci. Math. Hungar. 18 (1983), no. 2-4, 165-171.

[3] K. Böröczky and L. Szabó, Arrangements of 14, 15, 16 and 17 points on a sphere, Studia Sci. Math. Hungar. 40 (2003), no. 4, 407-421.

[4] L. Danzer, Finite point-sets on $S^{2}$ with minimum distance as large as possible, Discrete Math. 60 (1986), 3-66. 
[5] L. Fejes Tóth, Research Problems: Exploring a Planet, Amer. Math. Monthly 80 (1973), no. 9, 1043-1044.

[6] _Lagerungen in der Ebene auf der Kugel und im Raum, Springer-Verlag, Berlin-New York, 1972.

[7] _ Ü̈ber eine Abschätzung des kürzesten Abstandes zweier Punkte eines auf einer Kugelfläche liegenden Punktsystems, Jber. Deutsch. Math. Verein. 53 (1943), 66-68 (German)

[8] L. Hárs, The Tammes problem for $n=10$, Studia Sci. Math. Hungar. 21 (1986), no. 3-4, 439-451.

[9] J. Linhart, Eine extremale Verteilung von Grosskreisen, Elem. Math. 29 (1974), 57-59 (German).

[10] O. R. Musin and A. S. Tarasov, The Tammes Problem for $N=14$, Exp. Math. 24 (2015), no. $4,460-468$.

[11] _ The strong thirteen spheres problem, Discrete Comput. Geom. 48 (2012), no. 1, $128-141$.

[12] R. M. Robinson, Arrangements of 24 points on a sphere, Math. Ann. 144 (1961), 17-48.

[13] V. Rosta, An extremal distribution of three great circles, Mat. Lapok 23 (1972), 161-162 (1973) (Hungarian).

[14] K. Schütte and B. L. van der Waerden, Auf welcher Kugel haben 5, 6, 7, 8 oder 9 Punkte mit Mindestabstand Eins Platz?, Math. Ann. 123 (1951), 96-124 (German).

[15] P. M. L. Tammes, On the origin of number and arrangement of the places of exit on pollen grains, Rec. Trv. Bot. Neerl 27 (1930), 1-84.

[16] B. L. van der Waerden, Punkte auf der Kugel. Drei Zusätze, Math. Ann. 125 (1952), 213-222 (German).

Department of Geometry, Bolyai Institute, University of Szeged, Aradi vértanúk TERe 1, H-6720 Szeged, Hungary

E-mail address: fodorf@math.u-szeged.hu

Department of Geometry, Bolyai Institute, University of Szeged, Aradi vértanúk TERe 1, H-6720 Szeged, Hungary

E-mail address: vigvik@math.u-szeged.hu

Department of Geometry, Bolyai Institute, University of Szeged, Aradi vértanúk tere 1, H-6720 Szeged, Hungary

E-mail address: tzarnocz@math.u-szeged.hu 